\title{
ROTI maps of Greenland using kriging
}

Beeck, S. S.; Jensen, A. B. O.

Published in:

Journal of Geodetic Science

Link to article, DOI:

10.1515/jogs-2020-0123

Publication date:

2021

Document Version

Publisher's PDF, also known as Version of record

Link back to DTU Orbit

Citation (APA):

Beeck, S. S., \& Jensen, A. B. O. (2021). ROTI maps of Greenland using kriging. Journal of Geodetic Science, 11(1), 83-94. https://doi.org/10.1515/jogs-2020-0123

\section{General rights}

Copyright and moral rights for the publications made accessible in the public portal are retained by the authors and/or other copyright owners and it is a condition of accessing publications that users recognise and abide by the legal requirements associated with these rights.

- Users may download and print one copy of any publication from the public portal for the purpose of private study or research.

- You may not further distribute the material or use it for any profit-making activity or commercial gain

- You may freely distribute the URL identifying the publication in the public portal

If you believe that this document breaches copyright please contact us providing details, and we will remove access to the work immediately and investigate your claim 


\section{ROTI maps of Greenland using kriging}

DOI: https://doi.org/10.1515/jogs-2020-0123

Received January 17, 2021; accepted July 13, 2021

Abstract: GNSS satellite signals are affected by the media when traversing Earth's atmosphere, and the performance of GNSS based positioning and navigation is correlated with these effects. In the ionosphere, the signals are affected by the number of electrons along the signal path which can be quantified by the total electron content. The focus of this article is scintillation effects caused by electrons in the Arctic ionosphere, and the rate of the total electron content index, the ROTI, is used as a measure of the scintillation.

Data from permanent GNSS reference stations in Greenland is used to generate maps of the ROTI in Greenland, and the novelty of the work in this paper is the application of the ordinary kriging method as the basis for ROTI maps in the Arctic. Further, the choice of satellite constellations as well as the elevation mask used in the data analyses are analyzed and discussed. Also, the performance of using ordinary kriging for ROTI maps during times with both a low and a very high geomagnetic activity are discussed. The results show that ordinary kriging performs well during high geomagnetic activity, while during low activity the natural neighbor interpolation method is a better choice for the Arctic.

Keywords: Arctic, Global Navigation Satellite System, Greenland, Interpolation, Ionosphere, Kriging, Navigation, Rate of TEC index, Scintillation, Total Electron Content

\section{Introduction}

Satellite signals of the global navigation satellite systems (GNSS) are affected by the medium while being transmitted through Earth's atmosphere. The effect of the ionosphere is related to the amount of electrons along the signal path quantified by the total electron content (TEC), and the effect is dispersive for radio waves.

*Corresponding Author: S. S. Beeck: National Space Institute, Technical University of Denmark (DTU Space), 2800 Kongens Lyngby, Denmark, E-mail: saschu@space.dtu.dk

A. B. O. Jensen: Division of Geodesy and Satellite Positioning, KTH Royal Institute of Technology, Stockholm, Sweden
When using GNSS for geodetic applications, and other applications requiring a low uncertainty in the positioning, the effect of the ionosphere must be accounted for in the positioning process. The more knowledge we have about the effects, the better we are able to develop methods for handling the effects in the positioning. This applies not only to GNSS based positioning and navigation but also to other types of satellite communication where signals are affected during transmission through the ionosphere.

The effects of the ionosphere are enhanced, and thereby more challenging to handle, in the Equatorial and the Polar regions.

This paper focuses on the scintillation effects of the Arctic ionosphere, using data from GNSS stations in Greenland.

As a measure of scintillation, we focus on the rate of TEC index (ROTI), and the novelty of this paper is the application of the ordinary kriging method to the development of ROTI maps based on GNSS data from Greenland. Kriging is chosen based on a study (Durgonics, 2017) which identifies it as the best interpolation method for the ionosphere above the Iberian Peninsula in comparison with four other interpolation methods.

The purpose of the work is to take a step towards development of a space weather warning system for users of satellite communication in the Arctic, where users may be warned in case of increased ionosphere activity, which may reduce the quality of satellite communication, and in the worst cases prevent GNSS-based positioning and navigation (Khazanov, 2019) (p. 375). The results of the work described in this paper show that ordinary kriging is a useful interpolation method for ROTI maps during enhanced scintillation activity.

\subsection{The rate of TEC index (ROTI)}

The ionosphere is the ionized top layer of the Earth's atmosphere and it is generally considered to begin at a height of $60 \mathrm{~km}$ above the surface of the Earth. The ionization is mostly caused by the Sun, and the solar wind can generate great variation in the amount and the location of free electrons. As mentioned, a common measure of the amount of free electrons is the total electron content (TEC) which is the number of electrons in a column with a cross section 
area of one square meter, generally measured in TEC units (TECU) which is $10^{16}$ electron $/ \mathrm{m}^{2}$ (Klobuchar, et al., 1973) (Pi, et al., 1997).

Scintillations are fluctuations in the phase and amplitude of radio signals. They are caused by small scale variations in the electron content of the ionosphere as described by for instance (Aarons, 1982) (Yeh \& Liu, 1982). These variations in the electron density cause fluctuations in the refractive index which results in a diffraction of the signal. This can cause phase fluctuations, and the resulting interference between scattered signals causes amplitude scintillation (Hobiger \& Jakowski, 2017)(p. 183) (Linty, et al., 2018).

Phase scintillation can affect GNSS receivers by introducing cycle slips, where the continuous phase lock is lost momentarily. Cycle slips can be harmful to carrier phasebased GNSS positioning because the integer ambiguities must be re-estimated for continued positioning. The amplitude scintillations can result in a decrease of signal to noise ratio. If the signal to noise ratio fades to below a certain threshold it can result in a loss of lock as the GNSS receiver is no longer able to track the signal (Khazanov, 2019)(p. 374). In case of severe scintillation GNSS-based positioning and navigation may therefore not be possible for shorter or longer periods of time.

The amount of scintillation is often estimated by tracking of GNSS data in permanent GNSS stations, using the phase scintillation index $\sigma_{\varphi}$ or the amplitude scintillation index S4. However, these indices are typically computed with a sampling frequency in the GNSS receiver of at least $50 \mathrm{~Hz}$ (Durgonics, 2017) and this is not feasible with most GNSS stations available.

Since scintillation is co-located with strong gradients in the TEC (Mitchell, et al., 2005), the rate of TEC index (ROTI) is being used increasingly as an indication of the amount of scintillation. The ROTI is based on the standard deviation of the rate of TEC (ROT) (Pi, et al., 1997), and the index can be estimated from GNSS receivers operating with a sampling frequency of $1 \mathrm{~Hz}$ which is used in many GNSS reference stations.

The rate of change in TEC (ROT) can be computed as below (Kervalishvili, et al., 2018).

$$
R O T_{m}=\frac{T E C_{m}^{i}-T E C_{m-1}^{i}}{t_{m}-t_{m-1}}
$$

Where tis the time, $i$ is the satellite index and $m$ is the time index. From the ROT the ROTI can be computed as the standard deviation of ROT over a limited time period. Formula 2 is a slightly altered version of the ROTI formulas in (Ker- valishvili, et al., 2018) and (Jacobsen, 2014).

$$
\text { ROTI }_{n}=\sqrt{\frac{1}{N-1} \sum_{m=n}^{m+N-1}\left(R_{m} T_{m}-R \bar{O} T\right)^{2}}
$$

where $N$ is the number of ROT values used to calculate one ROTI value, $n$ is the ROTI time index, and $m$ is the ROT time index. $R \bar{O} T$ is the mean of ROT values in the time period used for the implementation.

Since 2017, ROTI maps have been an International GNSS Service product (Cherniak, et al., 2018). The International GNSS Service (IGS) maps are based on estimation of the TEC in ionospheric pierce points (IPPs) using a thin shell ionosphere model. Average ROTI values are calculated for each $2^{\circ} \times 2^{\circ}$ grid cell of the map if more than 30 values are available within the cell. The cell is left empty if less observations are available (Cherniak, et al., 2014). In this paper, we apply ordinary kriging as a new method for the spatial interpolation of ROTI maps for the Arctic region.

\subsection{Scintillations at high latitudes}

In the high latitude region, where Greenland is located, scintillation is primarily caused by increased particle precipitation in the auroral region or by high-speed convection of plasma patches. Both processes are increased during geomagnetic storms (Pi, et al., 1997), and also during sub storms (Meeren, et al., 2015).

Geomagnetic storms generally occur in relation to interplanetary coronal mass ejections (ICMEs) or corotating interaction regions (CIRs), which interacts with the Earth's magnetic field. Sub storms occur as a consequence of a sudden relaxation of the stretched arrangement of magnetic field lines in the magnetotail (Khazanov, 2019)(p. 60). This stretching and buildup of excess magnetic flux in the magnetotail is a result of a continuously negative $\mathrm{z}$ component of the interplanetary magnetic field (IMF) (Bz) for about an hour or less (Khazanov, 2019) (p. 59).

These processes cause the small-scale variations in the electron density in the ionosphere, which in turn affects radio signals traversing the ionosphere by producing scintillation (Hunsucker \& Hargreaves, 2003) (p.249). The effects of scintillation on GNSS signals, can be estimated at the discrete locations of GNSS receiver stations. To obtain an overall picture of the amount and the distribution of scintillation in Greenland, ROTI maps can be produced by combining the discrete observations with spatial interpolation methods. In this work, the geostatistical interpolation method, ordinary kriging, is used, and it is therefore introduced below. 


\subsection{Ordinary kriging}

Ordinary kriging is a geostatistical interpolation method which can be used when there is a locally constant but unknown mean field (Nielsen, 2009). It is a two-step procedure; first coefficients for a semi-variogram are estimated then the kriging (the actual interpolation) is carried out. For a thorough introduction to kriging see e.g. (Journel \& Huijbregts, 1978). Ordinary kriging has previously been used with good results in VTEC modelling (Mukesh, et al., 2020) and for scintillation mapping (Geng, et al., 2020).

With ordinary kriging, the estimated value in a point is computed by a linear combination of the values in the data points. The weights are computed to minimize the estimation variance and to be unbiased. Hereby, the mean of the estimations will be the same as the mean of the data points. Weights are estimated using the so-called semivariogram.

An empirical semi-variogram is based on the data points. The distance and semi-variance are calculated between all pairs of observation points. The data pairs and their corresponding semi-variance are then sorted into bins based on their inter-distance. The average semivariance is calculated for each bin and can be plotted with semi-variance on the y-axis and distance on the $\mathrm{x}$-axis (Nielsen, 2009), see for example Fig. 2

If second order stationarity is assumed, the semivariance function can be written as below, which is inspired by (Stein, 1999) and (Nielsen, 2009):

$$
y(h)=\frac{1}{2 N} \sum_{h \approx h_{i j}}\left(Z\left(x_{i}\right)-Z\left(x_{j}\right)\right)^{2}
$$

where $N$ is the number of pairs in the bin, $x_{i}$ and $x_{j}$ are a set of positions of data points, $Z\left(x_{i}\right)$ and $Z\left(x_{j}\right)$ are the values in the data points, $h$ is the distance specific for the bin, and $h_{i j}$ is the distance between the two positions.

Second order stationarity means that there must be the same co-variance between any two randomly chosen points within the same distance interval. This has to be true over the entire interpolation area.

A model is then fitted to the semi-variogram. Different models exist and they each have advantages depending on the type of data. The most common are the exponential, spherical, gaussian, cubic, and spline model. The models can be sorted into two groups depending on their behavior near the known points. The gaussian, cubic and spline models have a parabolic behavior near the known points, while the exponential and spherical models have a linear behavior near the known points (Geng, et al., 2020). Therefore, the gaussian, cubic and spline models are interesting for ionospheric scintillation. The five models have been analysed for scintillation over Southern China by (Geng, et al.,2020), where it was concluded that the gaussian model was the best fit for their study.

The gaussian model is based on estimates of the range (R), nugget $\left(\mathrm{C}_{0}\right)$, and sill $\left(\mathrm{C}_{0}+\mathrm{C}_{1}\right)$ and can be expressed as (PyKrige Documentation):

$$
y(h)=C_{0}+C_{1}\left(1-\exp \left(-\frac{|h|^{2}}{\left(\frac{4}{7} R\right)^{2}}\right)\right)
$$

The range is the distance at which the measurements are no longer correlated, and it is seen as a plateau in the semi-variogram (see e.g. Fig. 2). The sill is closely related to the range since it is the semi-variance corresponding to the range in the semi-variogram. The nugget is the value where the semi-variogram model intersects with the yaxis, meaning that it is a measure of the noise in the data.

The continuous modeled semi-variogram is then used to determine the weights of the data points for each predicted point. As mentioned earlier, the weights should minimize the estimation variance. The Lagrange multiplier method is therefore utilized, and it provides the equation system below, written as in (Nielsen, 2009) but with semi variance instead of covariance.

$$
\begin{aligned}
& {\left[\begin{array}{ccccc}
y\left(h_{11}\right) & y\left(h_{12}\right) & \cdots & y\left(h_{1 n}\right) & 1 \\
y\left(h_{21}\right) & y\left(h_{22}\right) & \cdots & y\left(h_{2 n}\right) & 1 \\
\vdots & \vdots & \ddots & \vdots & \vdots \\
y\left(h_{n 1}\right) & y\left(h_{n 2}\right) & \cdots & y\left(h_{n n}\right) & 1 \\
1 & 1 & \cdots & 1 & 0
\end{array}\right]\left[\begin{array}{c}
w_{1} \\
w_{2} \\
\vdots \\
w_{n} \\
-\lambda
\end{array}\right]} \\
& =\left[\begin{array}{c}
y\left(h_{01}\right) \\
y\left(h_{02}\right) \\
\vdots \\
y\left(h_{0 n}\right) \\
1
\end{array}\right]
\end{aligned}
$$

where $\lambda$ is the Lagrange multiplier. The weights can be calculated by matrix inversion, and they are individual for each interpolated point. With large data sets, calculation of the weights can induce a long computing time. It is noted that the quality of the results obtained with ordinary kriging interpolation is dependent on the density and the distribution of the data points used.

\section{Method and Results}

\subsection{Greenland GPS Network (GNET)}

The data in this study is from the Greenland GPS Network (GNET) (Arctic DTU, 2018). This is a network of permanent GNSS stations along the coast of Greenland. At the 
Table 1. Geographic latitude and longitude of the chosen GNET stations

\begin{tabular}{ccc}
\hline Station & $\begin{array}{c}\text { Latitude, North } \\
{[\mathrm{deg}]}\end{array}$ & $\begin{array}{c}\text { Longitude, West } \\
{[\mathrm{deg}]}\end{array}$ \\
\hline THU2 & 76.54 & 68.83 \\
UPVK & 72.79 & 56.13 \\
QEQE & 69.25 & 53.52 \\
SISI & 66.93 & 53.67 \\
NUUK & 64.18 & 51.73 \\
QAQ2 & 60.72 & 46.05 \\
SCO2 & 70.49 & 21.95 \\
\hline
\end{tabular}

time of writing there are 58 stations which are owned by the Agency of Data Supply and Efficiency which is a government organization in Denmark. Data from the stations can be obtained from this organization free of charge. DTU Space is responsible for the development, operation, and maintenance of the stations. The GNET stations have been established for geodynamic purposes, i.e. to study land movement due to ice melting of the glaciers in Greenland. Most stations operate with a data sampling frequency of $1 / 30 \mathrm{~Hz}$ which is sufficient for most geodynamic purposes. However, a few of the stations currently provide $1 \mathrm{~Hz}$ data. Out of these stations, only those with high-rate internet connection can provide GNSS-data in near real-time for investigation of the ionosphere above Greenland - currently that are the stations in Thule (THU2), Upernavik (UPVK), Qeqertalik (QEQE), Sisimiut (SISI), Nuuk (NUUK), Qaqortoq (QAQ2), and Scoresbysund (SCO2). The geographic latitude and longitude of the stations can be seen in Table 1. At some of the locations more than one GNSS receiver is installed and for the work carried out in this study, at locations with two or more $1 \mathrm{~Hz}$ stations, the receiver tracking both GPS and GLONASS was chosen. All except one of these stations are located on the west coast of Greenland, resulting in a slightly degraded data coverage on the east coast.

\subsection{Data period}

In this study, the analyses are focused on data from the days number 30 to 59 in the year 2014. The reason being that during February 2014 geomagnetically quiet as well as geomagnetically active periods occurred, providing a good basis for comparisons. Another reason is, that a geomagnetic storm which occurred on day 50 (the 19th of February 2014) has previously been investigated using data from Greenland (Durgonics, 2017). Comparing results of the current study with the previous study provides a way of validating the method.
We examined the geomagnetic activity during the investigation period by means of geomagnetic indices. The Auroral Electrojet (AE) index (Meng, et al., 2004) (Vennerstrøm, et al., 1991) is relevant since this study is concerned with the high latitude ionosphere. Day 50 of 2014 experienced a high AE of $1198 \mathrm{nT}$. Also, the Disturbance Storm Time Index (Dst) was investigated, and we identified day 50 as the day with the strongest storm of the period, resulting in the most negative Dst index of 2014 with a value of $-119 \mathrm{nT}$. Both a geomagnetically active and quiet time period should be considered as the scintillation processes differ. The other day of focus in this study is day 30, which is a geomagnetically quiet day, where the maximum $\mathrm{AE}$ index of the day is $185 \mathrm{nT}$ and the minimum Dst index is $-15 \mathrm{nT}$. The AE index during the period of day 30-59 of 2014 can be seen in Fig. 1

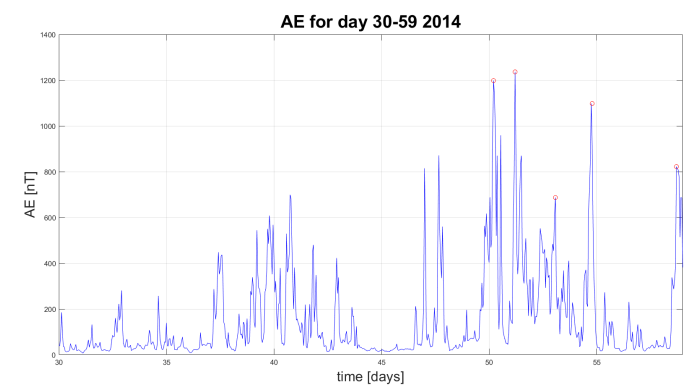

Fig. 1. AE data from day 30-59 of 2014. The storm events that are moderate or stronger are marked by red circles.

\subsection{Comparison of GPS and GLONASS}

Before investigating the performance of using ordinary kriging for ROTI mapping in Greenland, we carried out some preliminary analyses on the use of GPS and GLONASS as well as on the choice of elevation mask. These analyses are discussed in the following.

For this purpose, we used data from the receivers of the GNET-stations THU2, UPVK, NUUK, and QAQ2 which are all tracking both GPS and GLONASS. They are located at different geographic latitudes which can be seen in Table 1. For these stations, we investigated what the effect on the estimated vertical TEC (VTEC) would be, if GLONASS was included along with the GPS data. In this case, the VTEC values above the stations were analysed instead of the slant TEC, because the satellite signals do not take the same path through the ionosphere, and the slant TEC values would therefore not be comparable between the GNSS constellations. The VTEC values are calculated for each 
Table 2. The mean and standard deviation of the difference in VTEC when using GPS only versus both GPS and GLONASS. The differences are taken for the period of day 30 to 59 of 2014.

\begin{tabular}{ccc}
\hline Station & Mean difference [TECU] & $\begin{array}{c}\text { St. dev. of difference } \\
\text { [TECU] }\end{array}$ \\
\hline THU2 & -0.32 & 0.93 \\
UPVK & -0.11 & 1.07 \\
NUUK & 0.46 & 1.16 \\
QAQ2 & 0.21 & 0.81 \\
\hline
\end{tabular}

station using the program absolTEC from GNSS-lab which applies a mapping function to estimate the VTEC at the stations (Yasyukevich, et al., 2015) (Mylnikova, et al., 2015) (Yasyukevich, et al., 2015).

With absolTEC it is possible to choose an elevation cutoff angle, the time steps, and a correction coefficient. We set the elevation cut-off angle to 10 degrees, the time step to 0.05 hours, which is 3 minutes, and the correction coefficient which is a coefficient of the zenith angle to 0.94 , because this is the recommended value for high latitudes provided by the developers.

Using the full period of data from day 30 to day 59 of 2014, we computed the differences at the four GNSSstations between VTEC based on GPS only and VTEC based on both GPS and GLONASS. The mean and standard deviations of the differences can be seen in Table 2. The differences between the two sets of results generally have a mean very close to zero, indicating that there was no offset of importance. Therefore, we could include the IPPs from the GLONASS satellites without expecting an offset between the estimated GPS and GLONASS TEC results. This conclusion is supported by the values of the standard deviations of the differences, which are all small i.e. between 0.8 and 1.2 TECU.

\subsection{ROTI computation}

We then computed the ROTI values in each IPP. An IPP is the point where the satellite signal crosses a specific ionospheric height (Schaer, 1999), in this study it is set to $350 \mathrm{~km}$.

The ROTI values are calculated with time steps of 1 second over a 1-minute interval. This means that in our case $t_{m}-t_{m-1}=1$ second in Eq. (1), and $N=60$ in Eq. (2). However, $N$ is not always 60 since data points with elevation angles below the cut-off angle are eliminated. Therefore, it has been decided, in this study, that $N$ has to be at least 50, otherwise there is not enough data within the time window of one minute. So, for time epochs with $N$ less than 50 , the ROTI value for that minute is not calculated.
The method that we used to compute the ROTI values is similar to the method used in (Durgonics, 2017) and (Jacobsen, 2014). For verification purposes, ROTI computations based on $1 \mathrm{~Hz}$ data from GPS satellite G05 of the SCOR GNSS-receiver from 11:30 to 16:00 hours on day 50 of 2014, were compared with results from (Durgonics, 2017). This revealed that the peaks and the overall structure of the results are similar, and it becomes clear from the comparison that there is no apparent offset between the two methods applied.

\subsection{Elevation mask}

The reason why an elevation cut-off mask is needed, is that signals, from GNSS satellites with low elevation angles, have a longer path through the ionosphere. High ROTI values at low elevation angles are therefore more apparent on geomagnetically quiet days than on active days, because a larger fraction of the high ROTI values is caused by low elevation on quiet days. This can be verified by calculating the correlation between ROTI and elevation angle for both a geomagnetically active and quiet time. We did this for day 30 and 50 at selected epochs in time using both GPS and GLONASS. The correlations can be seen in Table 3. On day 30 the correlations are around -0.43 while they are significantly lower on day 50, indicating that some of the high ROTI values on the geomagnetically quiet day 30 may be due to signals at low elevation angle. The correlation at day 30 is overall constant, while the correlation at day 50 is slightly more fluctuating but consistently lower than for day 30 .

Based on these analyses we decided to use an elevation mask of 15 degrees. Some studies use higher elevation angles (Yang \& Morton, 2020) (Geng, et al., 2020) (Pi, et al., 1997), it could therefore be worth investigating if a higher elevation cut-off angle yields better results. However, in this study the network of GNSS-stations used is very sparse and therefore a higher elevation mask is not preferable, since 10-15 IPPs disappear for every step of five degrees,

Table 3. Correlation between the ROTI values and elevations angles at five different times

\begin{tabular}{ccc}
\hline & \multicolumn{2}{c}{$\begin{array}{c}\text { Correlation between ROTI and } \\
\text { elevation angle }\end{array}$} \\
\hline Time [UTC] & Day 30 of 2014 & Day 50 of 2014 \\
\hline 05:51:25 & $-\mathbf{0 . 4 1}$ & -0.19 \\
05:56:25 & -0.43 & -0.18 \\
06:01:25 & -0.43 & -0.04 \\
14:00:00 & -0.44 & -0.09 \\
16:00:00 & -0.52 & -0.23 \\
\hline
\end{tabular}


when the elevation cut-off angle is raised from 15 up to 35 degrees. An increase in cut-off angle will therefore significantly decrease the number of IPPs which is already low because of the sparse GNSS network in Greenland used in this study. Raising the elevation mask could, however, be beneficial in cases with more GNSS stations and thereby better coverage with more IPP data points. Further investigations of the coverage can be found in (Beeck, 2020).

The elevation mask of 15 degrees is higher than the elevation mask used for the analysis in section 2.3, however the results from section 2.3 are still valid, since we are simply reducing the number of IPPs rather than adding more.

\subsection{Spatial interpolation}

Five different methods of interpolation of TEC were tested in (Durgonics, 2017) (pp. 52-57). The methods are inverse distance weighting, kriging, natural neighbor, spline, and trend interpolation. It was found that the methods which provide most details of small-scale variations are kriging and the natural neighbor methods (Durgonics, 2017) (pp. 54-57). Kriging was identified as the optimal method because the root mean square of the interpolation residuals is a bit lower than for the natural neighbor interpolation (Durgonics, 2017) (p. 55). It should be mentioned that the study was performed based on TEC data from the Iberian Peninsula. This introduces two primary differences between the study in (Durgonics, 2017) and this current study. First, it means that the other study was done in the mid latitudes, and the mid latitude ionosphere does not contain the same phenomenon's as the high latitude ionosphere. Secondly, it was done in a region with more GNSS stations and also the stations are much more regularly spaced than in Greenland. Nonetheless, it is assumed that these differences do not change the fact that kriging and natural neighbor are the best interpolation methods. Especially since the IPPs, although being relatively few in numbers, do cover most of Greenland. The spatial distributions of the IPPs for some of the epochs analysed are shown in for instance Fig. 3, Fig. 4, and Fig. 5.

In this study we tested ordinary kriging first, since it was found to be the best interpolation method used with the data set from the Iberian Peninsula in (Durgonics, 2017), and because to our knowledge it has not previously been tested with data from the Arctic or Greenland. Afterwards, we tested natural neighbor interpolation of the ROTI values and compared it to the ordinary kriging method as discussed in the following.

The ordinary kriging interpolation was performed in Python with the package PyKrige, the documentation for the package can be found in (PyKrige Documentation). A fixed set of parameters are used for all ordinary kriging interpolated maps, since that assures a common statistical basis. Fixed parameters assume that the noise is proportional to the signal, and that the range is constant over time. Also, second order stationarity is assumed temporally. This might however only be reasonable over short time scales, and both short- and long-time intervals were therefore tested.

First, we examined the empirical semi-variograms of day 30 and day 50 in 2014 to get an idea of what the parameters should be. The signal on day 30 was under the noise level, and it was therefore concluded that the data from day 30 , which is a geomagnetically quiet day, is not appropriate for choosing the ordinary kriging parameters. It could also be argued that the parameters should be based on a time of high geomagnetic activity, since scintillation maps might be of higher interest to end users during such days.

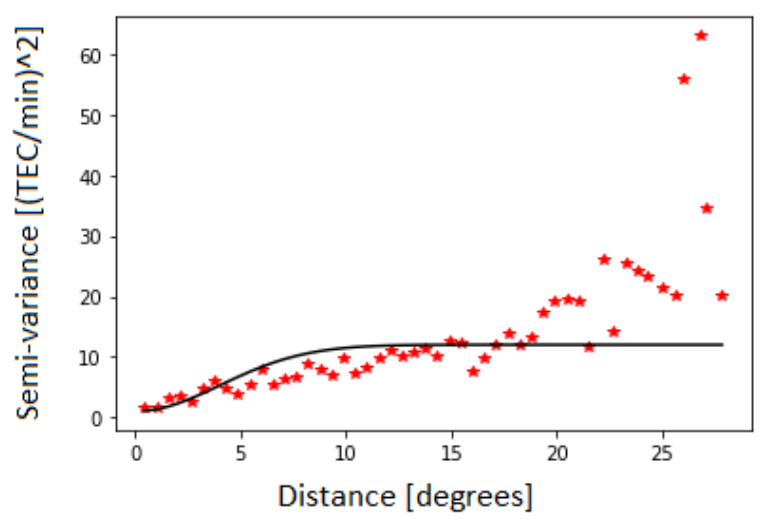

Fig. 2. Semi-variogram calculated for day 50 at 05:51:25 UTC, with sill $=12(\mathrm{TECU} / \mathrm{min})^{2}$, range $=10$ degrees and nugget $=1$ $(\mathrm{TECU} / \mathrm{min})^{2}$.

The semi-variogram for day 50 is shown in Fig. 2. Figure 2 shows a low semi-variance for data points close to each other, and it increases for data points with larger distance between the points. In the calculations, spherical distances were used and the unit of distance in the $\mathrm{x}$ axis is therefore degrees. The authors chose the Gaussian model since this was visually the best fit to the empirical semi-variogram. It was also concluded in (Geng, et al., 2020) for a study area in Southern China, that the Gaussian model matches the semi-variogram best. The parameters sill $=12(\mathrm{TECU} / \mathrm{min})^{2}$, range $=10$ degrees, and nugget $=1(\mathrm{TECU} / \mathrm{min})^{2}$ turn out to be the best fit to the empirical semi-variogram with the current data set from Greenland. 
The semi-variance for distances longer than the range should generally be around the sill. In Fig. 2 there are some values at large distances which have a very high semivariance. This is because there are few data-pairs in these bins. For all the ROTI maps discussed in the following, the semi-variogram of Fig. 2 has been used.

The next step is to perform the actual interpolation using the ordinary kriging method to obtain a ROTI map. Fig. 3 shows a ROTI map of Greenland for 05:51:25 hours UTC on day 50 of 2014.

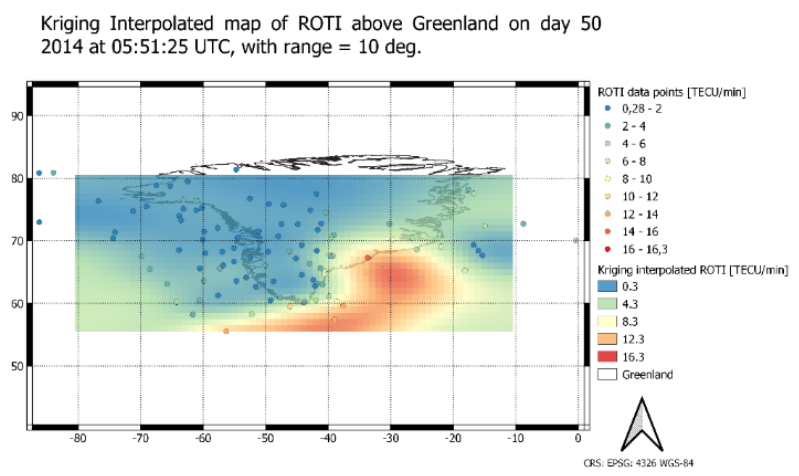

Fig. 3. Ordinary kriging interpolated ROTI map on day 50 at 05:51:25 UTC, with sill $=12(\mathrm{TECU} / \mathrm{min})^{2}$, range $=10$ degrees, and nugget $=1$ $(\mathrm{TECU} / \mathrm{min})^{2}$.

The extrema of the interpolation in Fig. 3 fits well with the extrema of the data. For day 50 at 05:51:25 UTC we used the parameters; sill $=12(\mathrm{TECU} / \mathrm{min})^{2}$, range $=10$ degrees, and nugget $=1(\mathrm{TECU} / \mathrm{min})^{2}$ with good results. The authors therefore investigated whether these parameters can also be used for later epochs in time of the same day. The chosen time epochs were five and ten minutes after the initial map, supplemented by maps from 14:00:00 UTC and 16:00:00 UTC. We chose these epochs in time so that various time intervals are tested. The figures are set to have similar color scale in order to make the maps comparable.

The ROTI map in Fig. 4 is a snap shot of the ionospheric scintillation five minutes after Fig. 3. The ROTI values are still high, even though they are lower than in Fig. 3. The very high ROTI values in the south eastern part of the map are not present here. However, in Fig. 5 it is seen that five minutes later at 06:01:25 UTC the ROTI values increase again for this area, and the plot becomes similar to the values at 05:51:25 UTC. The reason for the lower ROTI values at 05:56:25 UTC might just be a different spatial distribution of the IPPs, since this ROTI structure is based on relatively few data points.

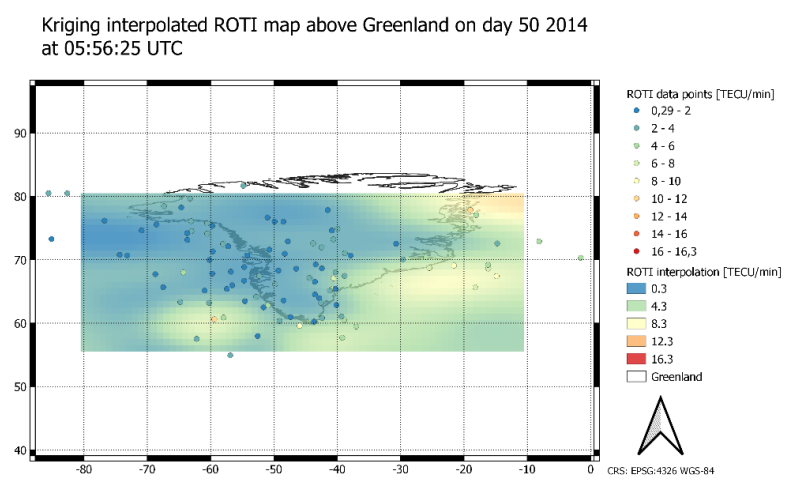

Fig. 4. Ordinary kriging interpolated ROTI map on day 50 at 05:56:25 UTC, with sill $=12(\mathrm{TECU} / \mathrm{min})^{2}$, range $=10$ degrees, and nugget $=1$ $(\mathrm{TECU} / \mathrm{min})^{2}$.

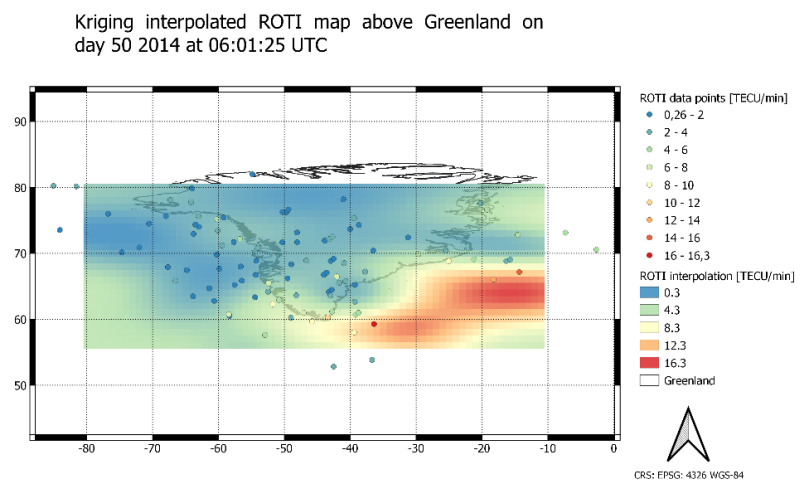

Fig. 5. Ordinary kriging interpolated ROTI map on day 50 at 06:01:25 UTC, with sill $=12(\mathrm{TECU} / \mathrm{min})^{2}$, range $=10$ degrees, and nugget $=1$ $(\mathrm{TECU} / \mathrm{min})^{2}$.

Fig. 6 is based on data from day 50 at 14:00:00 UTC i.e. approximately eight hours later on the same day as Fig. 3. The scintillation activity illustrated by the ROTI values has obviously decreased during the day, but some of the ROTI values are in the range 4-8 TECU/min which are still high values.

Both Fig. 6 and Fig. 7 have interpolation extrema that are within the data range, and both interpolations generally reflect the variations in the data well. The ROTI values in Fig. 7 are a bit higher than in Fig. 6, but not much, and it still shows an ionosphere with a lower level of scintillation than in Fig. 3, Fig. 4 and Fig. 5.

One of the advantages of the kriging method is that the estimation variance can be computed and plotted together with the estimate. One example is seen in Fig. 8 where the square root of the estimation variance is shown 
Kriging interpolated ROTI map above Greenland on day 50 at 14:00:00 UTC

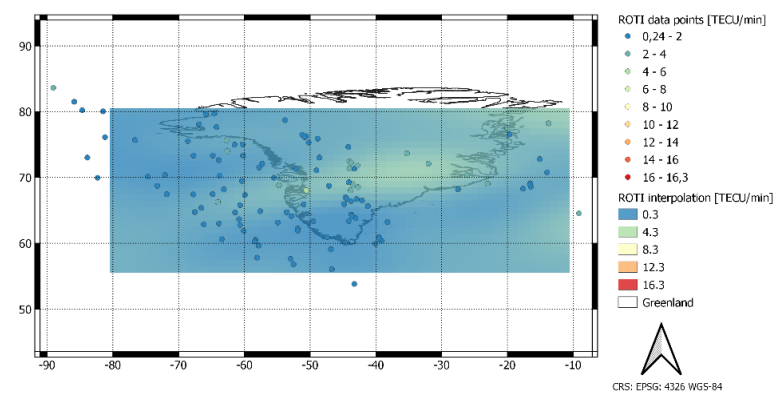

Fig. 6. Ordinary kriging interpolated ROTI map on day 50 at 14:00:00 UTC, with sill $=12(\mathrm{TECU} / \mathrm{min})^{2}$, range $=10$ degrees, and nugget $=1(\mathrm{TECU} / \mathrm{min})^{2}$.

Kringing interpolated ROTI map above Greenland on day 502014 at 16:00:00 UTC
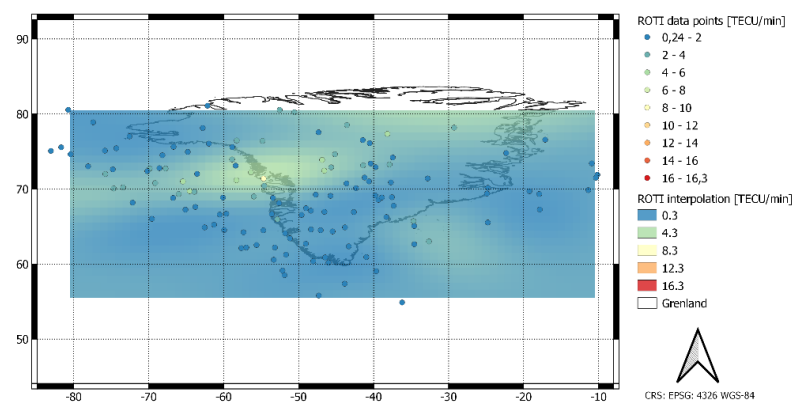

Fig. 7. Ordinary kriging interpolated ROTI map on day 50 at 16:00:00 UTC, with sill $=12(\text { TECU } / \mathrm{min})^{2}$, range $=10$ degrees, and nugget $=1(\mathrm{TECU} / \mathrm{min})^{2}$.

Standard deviation of the kriging estimate of ROTI above Greenland on day 502014 at 05:51:25 UTC

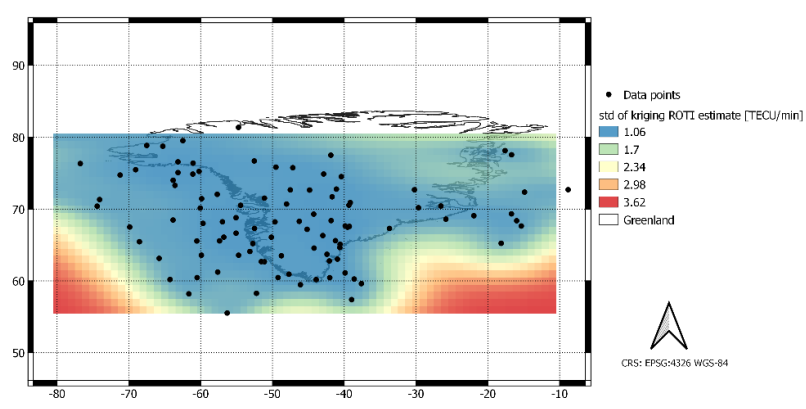

Fig. 8. Standard deviation map of the ordinary kriging estimates above Greenland on day 502014 at 05:51:25 UTC above Greenland at 05:51:25 UTC. From Fig. 8 it is clear that the standard deviation of the ordinary kriging estimate is largest in the areas with the poorest data coverage. This is seen in Fig. 8 as a very clear edge of higher standard deviation where there is a transition from interpolation to extrapolation in the map.

The authors also performed ordinary kriging with data for day 30, but the ROTI values in the IPPs did not coincide well with the interpolated ROTI values. We therefore decided to use another interpolation method. The natural neighbor method is used with good results in the test with TEC data from the Iberian Peninsula as previously mentioned (Durgonics, 2017) (p. 55), and it was also tested for interpolation of VTEC from Greenland in (Durgonics, 2017)(p. 69). The natural neighbor method is therefore applied as an alternative method for interpolation of ROTI in this study.

We investigated natural neighbor interpolation at both geomagnetically quiet and active times. The reason for investigating also for geomagnetically active time periods is that the natural neighbor method is a computationally lighter method, and it is therefore interesting to examine the difference between the two interpolation methods. The natural neighbor interpolations of the ROTI are computed in QGIS, with the natural neighbor SAGA toolbox (QGIS).

Natural neighbour interpolated ROTI map above Greenland on day 302014 at 05:51:25 UTC

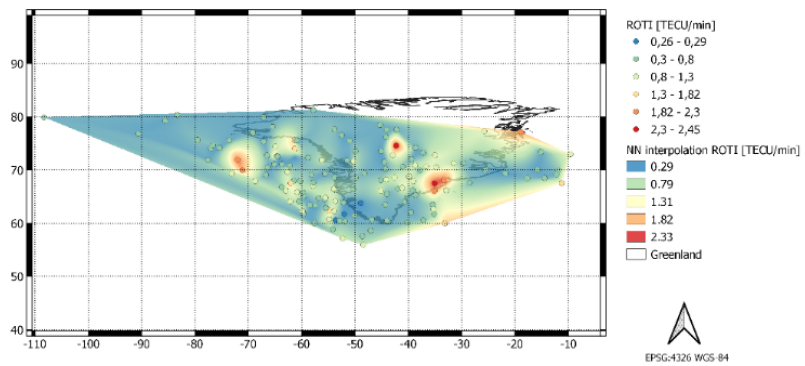

Fig. 9. Natural Neighbor interpolation of ROTI on day 30 at 05:51:25 UTC.

Figure 9 shows the natural neighbor interpolation of ROTI on day 30 at 05:51:25 UTC. The interpolated values are within the limits of the data, and the interpolation assumes the exact values in the data points. This is most relevant in situations where the measurements are extremely precise. If this is not the case then it might be better to have a higher degree of smoothing, otherwise some data points might produce peaks in the interpolation. This is 
seen in Fig. 9 as small-scale structures which are based on one to three data points. Structures based on only one data point should always be interpreted with caution. The same is true for the edges of the interpolation.

It can be very illustrative to see some of the characteristics of the two different interpolation methods in the figures. The natural neighbor method was therefore also tested for day 50, to investigate how the result deviates from the corresponding ordinary kriging-based map.

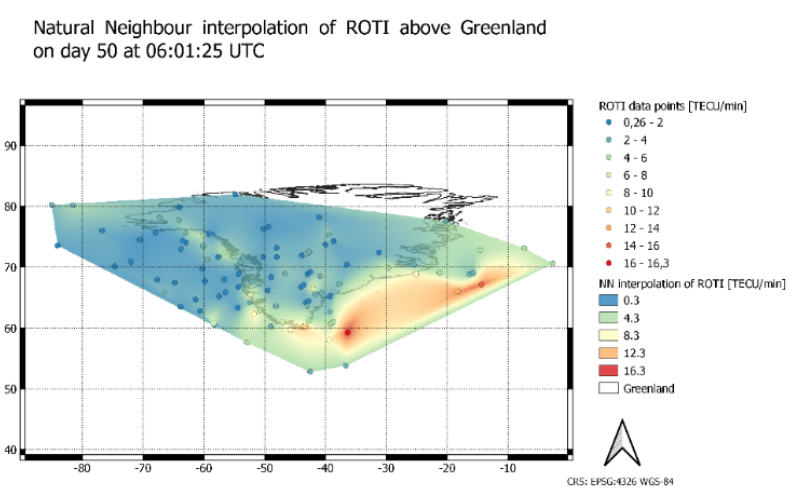

Fig. 10. Natural Neighbor interpolation of ROTI on day 50 at 06:01:25 UTC.

The natural neighbor interpolation in Fig. 10 is based on the same data as the ordinary kriging interpolation in Fig. 5. The general features of the two plots are similar, and the natural neighbor interpolation on day 50 exhibits a fine smoothness, but it is not as smooth as the corresponding ordinary kriging interpolation. This means that the map exhibits small scale structures based on single data points in Fig. 10. An increase in the number of data points could possibly reveal whether the one-point structures are a result of scintillation or measurement errors.

In Table 4 the mean and standard deviation of the difference between the two interpolation methods are shown for three separate times. Table 4 indicates that there is no apparent offset between the natural neighbor and kriging estimate. This is valuable information when using, and choosing between, the two methods. This shows the importance of utilizing both a statistical analysis, as seen in Table 4, and a visual analysis, as done with the plots above. The visual analysis has a focus on the peaks and smoothness around the individual points which can not be reflected in the statistical analysis, while the statistical analysis investigates the offset in a quantitative manner
Table 4. Mean and standard deviation of the difference between the natural neighbor interpolation and ordinary kriging estimate in 21 points at 05:51:25 and 05:56:25 UTC and 20 points at 06:01:25 UTC on day 502014 .

\begin{tabular}{lccc}
\hline & $05: 51: 25$ UTC & $05: 56: 25$ UTC & $06: 01: 25$ UTC \\
\hline $\begin{array}{c}\text { Mean of } \\
\text { difference }\end{array}$ & 0.19 & 0.11 & 0.00 \\
$\begin{array}{l}\text { [TECU } / \mathrm{min}] \\
\begin{array}{c}\text { St. dev. of } \\
\text { difference }\end{array}\end{array}$ & 0.78 & 1.14 & 1.32 \\
\hline [TECU/min] & & & \\
\hline
\end{tabular}

which would not be possible visually. The interpolation methods are further discussed in the following section.

\subsection{Validation of interpolated ROTI maps}

As a validation, the result shown in Fig. 3 is compared to a ROTI map from the Norwegian Mapping Authority, which can be found at (Norwegian Mapping Authority). They produce ROTI maps above Scandinavia every five minutes. These Scandinavian maps do not cover Greenland, but the interpolated ROTI values at the western edge of the Scandinavian maps, gives an idea of the ROTI values that should be seen on the eastern edge of a Greenland map as Fig. 3. The ROTI map in Fig. 3 agrees with the ROTI values in the corresponding ROTI map from the Norwegian Mapping Authority from the same epoch in time. It is noted that the ROTI maps from the Norwegian Mapping Authority are produced with the maximum value constrained to 5 TECU/minute (Offenbacher \& Thornström, 2020), and such constraint has not been applied in the ROTI maps for Greenland generated in this study.

Just as with Fig. 3, we used the ROTI maps from the Norwegian Mapping Authority for validation of the other interpolated ROTI maps produced in this study. The previous kriging plots all have values that approximately correspond to the ROTI values in the adjacent areas of the ROTI maps from the Norwegian Mapping Authority.

In Fig. 8 the standard deviation of the kriging estimate was provided as a measure of the uncertainty of the method. This is a reasonable result for our purpose, which is to test the kriging method in an attempt to get closer to a warning system of scintillation impact maps for Greenland. It is always a matter of the application and end-user need whether this reliability is sufficiently high. However, more GNSS stations at different locations in Greenland, and an inclusion of more GNSS satellite systems would increase the reliability of the ROTI maps. 


\section{Discussion}

Theoretically, ordinary kriging would be expected to be the best interpolation method especially in areas with suboptimal data coverage such as Greenland. This is because the ordinary kriging method is based on a statistical estimation that takes the data noise into consideration. Also, it provides a variance estimate which can be used for evaluation of the results. Other interpolation methods, such as natural neighbor, are deterministic and do not consider data noise. Even though the ordinary kriging method is a theoretically better method for this purpose, the natural neighbor method did, in this study, produce similar overall structures and reasonable smoothing in scintillation maps of day 50, despite some chimney-like structures based on single data points. Benefits of the natural neighbor interpolation method is that the computation time is generally shorter than for ordinary kriging, and no a priori parameters need to be chosen. However, the ordinary kriging method did produce smoother interpolations, while still reflecting the overall variations in the data on day 50. Further, it is a clear advantage to know the variance of the estimates as is produced with ordinary kriging.

Nonetheless, ordinary kriging was not optimal for day 30 where the ROTI values are very low. It might therefore be best to use natural neighbor interpolation at geomagnetically quiet periods in time. It could also be argued that if ROTI maps are to be used as part of a warning system, GNSS users will mostly be interested in scintillation information at geomagnetically active times, and that the maps should therefore only be produced at these times.

No matter which one of the two options are chosen it would require an activity parameter and a threshold to choose between the methods in an operational setup. The parameter must be available in near real-time, and it would therefore make sense to make use of data from GNSS Ionospheric Scintillation and TEC Monitors (GISTM) (Septentrio, 2016) as they can provide scintillation indices in near real-time. The problem is that the number of GISTM stations in Greenland is very limited. A combination of multiple parameters is also a possibility. The scintillation data could be complemented by geomagnetic data such as the Polar Cap North (PCN) index (Vennerstrøm, et al., 1991) which is available in near real-time (Technical University of Denmark, Arctic and Antarctic Research Institute). Another possibility is to include solar wind data which could also provide an opportunity of forecasting the geomagnetic activity. These topics are currently being investigated as part of new research projects at DTU Space.

\subsection{Future work}

For a real time monitoring of the ionosphere it would be beneficial to have GISTMs in Greenland, because such receivers calculate scintillation indices locally. Transmitting these indices instead of the raw GNSS data requires less data bandwidth. This is an advantage in near realtime monitoring, especially in Greenland, where the internet connection can be very limited, particularly on the east coast. There were only two GISTM, more specifically Septentrio PolaRx5S receivers, in Greenland at the time of this study, they are located at the QAQ2 and THU2 stations, for which the coordinates can be found in Table 1. The PolaRx5S receivers provide both $\sigma_{\varphi}$ and S4 indices in ionospheric scintillation monitoring (ismr) data files (Septentrio, 2016), but they do not calculate ROTI (Septentrio, 2016)(pp. 32-33). ROTI is well correlated to $\sigma_{\varphi}$, but not linearly (Durgonics, 2017) (p. 76) and it can therefore be challenging to compare the two indices. Furthermore, the TEC values in the ismr data files cannot be used for calculating ROTI, since the resolution is too sparse.

Considering a future scintillation warning system, one possible solution is to develop a program which can compute the ROTI values locally at the stations, and then transmit the ROTI data either separately or in the ismr file. Another solution is to install more GISTMs along the coast of Greenland, since the GNET $1 \mathrm{~Hz}$ data would then not be needed as a supplement. Hence the $\sigma_{\varphi}$ index could be used exclusively.

PolaRx5S receivers are used in both Canada (Canadian High Arctic Ionospheric Network) and Norway (Dr. K. S. Jacobsen, personal communication). Installing more PolaRx5S receivers or similar in Greenland might therefore make the comparison of data easier, and permit better opportunities for cooperation on monitoring of the high latitude ionosphere in the future.

As previously mentioned, solar wind parameters might be useful in deciding which interpolation method to use. Nonetheless, there are also other interesting possibilities for using solar wind parameters in future work. It is described in (Durgonics, 2017) (p. 128), (Jin, et al., 2019) and (Xiong, et al., 2019) that the y-component of the IMF influences at which magnetic local time the strongest scintillation will occur. This could potentially improve predictions of the spatial distribution of scintillation in the future.

In previous work done by the authors in using ordinary kriging for mapping of VTEC in Norway (Jensen, et al., 2007), investigations on the temporal update of the semivariogram showed that a four-hour update rate was suitable during medium ionosphere activity. This may not be 
the same for ROTI maps, but a similar investigation should be carried out to investigate a suitable temporal update rate for ROTI maps of Greenland.

Also, given the data availability, an upper limit for the range of the semivariogram should be investigated. For instance, with the data used in the study presented in this paper, it could be argued that the semivariogram should not be applied for a range larger than 20 degrees, since there are not enough data pairs above this range.

\section{Conclusion}

In this paper we have shown that ordinary kriging can be used for GNSS-based ROTI maps of Greenland during times with increased geomagnetic activity.

The spatial interpolations tested with ordinary kriging and natural neighbor both gave good results for geomagnetically active times. However, the ordinary kriging interpolation was smoother than the natural neighbor, while still reflecting the variability of the data. During geomagnetically quiet times the natural neighbor interpolation was preferred since it honored the values of the data points more than the ordinary kriging interpolation. The optimal approach therefore seems to be a solution where the interpolation method is chosen based on the amount of geomagnetic activity.

This paper shows, that it is possible to monitor the ionosphere above Greenland with data from the existing GNET stations. However, if monitoring shall be carried out in near real-time, it would be beneficial to install GISTMs in Greenland. If enough GISTMs are established, they can form the basis for an ionosphere monitoring system independent of other systems. New GISTMs will also increase the coverage by tracking data from the Galileo satellites along with data from GPS and GLONASS. This will increase the amount of GNSS data available and will also provide a better coverage of IPPs in Greenland.

Acknowledgements: Our colleagues Prof. Per Knudsen, Dr. Claus Sølvsteen, Søren Skaarup Larsen, and Dr. Susanne Vennerstrøm are all gratefully acknowledged for discussions and suggestions to this work. Finn Bo Madsen at DTU Space is acknowledged for helping with the GNETdata and discussions around the GNSS infrastructure in Greenland. Charlotte Havsteen at the Defence Acquisition and Logistics Organisation in Denmark is acknowledged for providing and supporting the idea to this study.
The Danish Agency for Data Supply and Efficiency (SDFE) is acknowledged for the GNET data.

\section{Abbreviations}

- Dst - Disturbance storm time index

- GISTM - GNSS Ionospheric Scintillation and TEC Monitor

- GLONASS - Global Navigation Satellite System

- GNET - Greenland GPS Network

- GNSS - Global Navigation Satellite System

- GPS - Global Positioning System

- IMF - Interplanetary Magnetic Field

- IPP - Ionospheric Pierce Point

- ismr - ionospheric scintillation monitoring, data format

- $\mathbf{n T}$ - nano Tesla

- ROT - Rate of TEC

- ROTI - Rate of TEC Index

- TEC - Total Electron Content

- TECU - TEC unit

- VTEC - vertical TEC

\section{References}

Arctic DTU, 2018, GNET GPS stations, https://arctic.dtu.dk/english/ research/research-infrastructure/gnet-gps-stations, Accessed 11-06-2021.

Beeck S., 2020, TEC and Scintillation in the lonosphere above Greenland. Master thesis, Technical University of Denmark. [3] Canadian High Arctic lonospheric Network, CHAIN - the Canadian High Arctic lonospheric Network, http://chain. physics.unb.ca/chain/pages/gps/\#PolaRxS, Accessed 1310-2020.

Cherniak I., Krankowski A. and Zakharenkova I., 2014, Observations of the ionospheric irregularities over the Northern Hemisphere: Methodology and service, Radio Sci., 49, 653-662.

Cherniak I., Krankowski A. and Zakharenkova I., 2018, ROTI maps: a new IGS ionospheric product characterizing the ionospheric irregularities occurrence, GPS solut, 22, 3, 69.

Durgonics T., 2017, Multi-Instrument Observations of Physical Processes in the Arctic lonosphere and Derived Applications. PhD thesis, Technical University of Denmark.

Geng W., Huang W., Liu G., Aa E., Liu S., Chen Y. and Luo B., 2020, Generation of ionospheric scintillation maps over Southern China based on Kriging method, Adv. Space Res., 65, 12, 28082820.

Hobiger T. and Jakowski N., 2017, Atmospheric Signal Propagation, In: Teunissen and Montenbruck (Eds.), Springer Handbook of Global Navigation Satellite Systems, Springer International Publishing.

Hunsucker R. D. and Hargreaves J. K., 2003, The High-Latitude lonosphere and its Effects on Radio Propagation, Cambridge University Press. 
Jacobsen K., 2014, The impact of different sampling rates and calculation time intervals on ROTI values, J. Space Weather Space Clim., 4, A33.

Jensen A., Øvstedal O. and Grinde G., 2007, Developement of a Regional lonosphere Model for Norway, Proceedings of the 20th International Technical Meeting of the Satellite Division of the Institute of Navigation- ION GNSS, 2880-2889.

Jin Y., Spicher A., Xiong C., Clausen L. B., Kervalishvili G., Stolle C. and Miloch W. J., 2019, Ionospheric Plasma Irregularities Characterized by the Swarm Satellites: Statistics at High Latitudes, J. Geophys. Res.-Space, 124, 2, 1262-1282.

Journel A. G. and Huijbregts C. J., 1978. Mining Geostatistics, Academic Press.

Kervalishvili G., Xiong C., Stolle C., Rauberg J. and IJsse J., 2018, Computation of TEC and Rate of TEC Index (ROTI) from GOCE GPS, Technical note prepared by GFZ German Research Centre for Geosciences and Delft University of Technology, Doc. No. GO-TN-HPF-GS-0337.

Khazanov G. W. (Ed.), 2019, Space Weather Fundamentals, CRC Press, Taylor and Francis Group.

Klobuchar J. A., Mendillo M., Seeman D. R., Aarons J., Allen R. S., Mullen J. P. and Basu S., 1973, Total electron content studies of the ionosphere, Air Force Cambridge Research Laboratories, technical report 73-0098, National Technical Information Service U.S. Department of Commerce.

Linty N., Minetto A., Dovis F. and Spogli L., 2018, Effects of phase scintillation on the GNSS positioning error during the September 2017 storm at Svalbard, Space Weather, 16, 9, 1317-1329.

Meeren C., Oksavik K., Lorentzen D. A., Rietveld M. T. and Clausen L. B., 2015, Severe and localized GNSS scintillation at the poleward edge of the nightside auroral oval during intense substorm aurora, J. Geophys. Res.-Space, 2015, 120, 12, 1060710621.

Meng C., Takahashi K., Kamei T., Kikuchi T. and Kunitake M., 2004, Near-Real-Time Auroral Electrojet Index: An International Collaboration Makes Rapid Delivery of Auroral Electrojet Index, Space Weather, 2, 11.

Mitchell C. N., Alfonsi L., De Franceschi G., Lester M., Romano V. and Wernik A. W., 2005, GPS TEC and scintillation measurements from the polar ionosphere during the October 2003 storm, Geophys. Res. Lett., 32.

Mukesh, R., Karthikeyan, V. and Sindhu, P., 2020, Ordinary kriging - and cokriging - based surrogate model for ionospheric TEC prediction using NavIC/GPS data, Acta Geophysica, 68, 5, 1529-1547.

Mylnikova A. A., Yasyukevich Yu. V., Kunitsyn V. E. and Padokhin A. M., 2015, Variability of GPS/GLONASS differential code biases, Results Phys., 5, 9-10.

Nielsen A. A., 2009, Geostatistics and Analysis of Spatial Data, Lecture Notes, Technical University of Denmark, Available at: https://www2.imm.dtu.dk/pubdb/edoc/imm5177.pdf.

Norwegian Mapping Authority, SeSolstorm, Available at: https: //sesolstorm.kartverket.no/moreplots.xhtml, Accessed 11-062021.

Offenbacher C. and Thornström E., 2020, lonospheric Models for GNSS Measurements. Bachelor thesis, Royal Institute of Technology in Stockholm.

Pi X., Mannucci A., Lindqwister U. and Ho C., 1997, Monitoring of Global ionospheric irregularities using the worldwide GPS network, Geophys. Res. Lett., 24, 2283-2286.
PyKrige Documentation, Available at: https://zenodo.org/record/ 3739879\#.Xu90oWgzZEY Accessed 21-09-2020.

QGIS, QGIS SAGA toolbox, Available at: https://docs.qgis.org/2. 8/en/docs/user_manual/processing_algs/saga/index.html, Accessed 11-06-2021.

Schaer S., 1999, Mapping and predicting the Earth's ionosphere using the Global Positioning System. PhD thesis, Société helvétique des sciences naturelles, Commision géodésique, Eidgenössische Technische Hochschule Zürich, Institut für Geodäsie und Photogrammetrie.

Septentrio, 2016, PolaRx5S User Manual

Stein M. L., 1999, Interpolation of Spatial data - Some Theory for Kriging, Springer Science+Business Media New York.

Technical University of Denmark, Arctic and Antarctic Research Institute, Real time Pocal Cap magnetic index, https://pcindex.org/, Accessed 18-09-2020.

Vennerstrøm S., Friss-Christensen E., Troshichev O. A. and Andresen V. G., 1991, Comparison between the polar cap index, $\mathrm{PC}$, and auroral electrojet indices $\mathrm{AE}, \mathrm{AL}$, and $\mathrm{AU}$, J. Geophys. Res.-Space, 96, A1, 101-113.

Xiong C., Yin F., Luo X., Jin Y. and Wan X., 2019, Plasma patches inside the polar cap and auroral oval: the impact on the spaceborn GPS receiver, J. Space Weather Space Clim., 9, A25.

Yang Z. and Morton Y. J., 2020, Time lags in Ionospheric Scintillation Response to Geomagnetic Storms: Alaska Observations, Proceedings of the ION GNSS+ 2020 VIRTUAL.

Yasyukevich Yu. V., Mylnikova A. A. and Polyakova A. S., 2015, Estimating the total electron content absolute value from the GPS/GLONASS data. Results Phys., 5, 32-33.

Yasyukevich Yu. V., Mylnikova A. A., Kunitsyn, V. E. and Padokhin A. S., 2015, Influence of GPS/GLONASS differential code biases on the determination accuracy of the absolute total electron content in the ionosphere, Geomagn. Aeronomy+, 55, 763769.

Yeh K. C. and Liu C. H., 1982, Radio wave scintillations in the ionosphere, In: Proceedings of the IEEE, 70, 4, 324-360.

Aarons J., 1982, Global morphology of ionospheric scintillations, In: Proceedings of the IEEE, 70, 4, 360-378. 